
allemande

51-1 | 2019

La République démocratique allemande dans l'espace public européen (1949-2018)

\title{
Comment les écrivains sorabes ont perçu la RDA
}

Jean Kudela

\section{(2) OpenEdition \\ Journals}

Édition électronique

URL : https://journals.openedition.org/allemagne/1578

DOI : $10.4000 / a l l e m a g n e .1578$

ISSN : 2605-7913

Éditeur

Société d'études allemandes

Édition imprimée

Date de publication : 2 juillet 2019

Pagination : 155-176

ISSN : 0035-0974

\section{Référence électronique}

Jean Kudela, "Comment les écrivains sorabes ont perçu la RDA », Revue d'Allemagne et des pays de langue allemande [En ligne], 51-1 | 2019, mis en ligne le 02 juillet 2020, consulté le 02 juin 2022. URL : http://journals.openedition.org/allemagne/1578 ; DOI : https://doi.org/10.4000/allemagne.1578 


\title{
Comment les écrivains sorabes ont perçu la RDA
}

\author{
Jean Kudela *
}

Les Sorabes ont été au VI ${ }^{\mathrm{e}}$ siècle une avant-garde slave qui a peu à peu occupé tout l'espace oriental et central actuel de l'Allemagne. La conquête franque aura dans un second temps réduit leur habitat à la province historique de Lusace. Il aura fallu aux Sorabes attendre un millénaire avant d'être reconnus comme peuple par un État allemand, par la RDA précisément. Les Sorabes y ont constitué une minorité supposée modèle définie selon les principes marxistes-léninistes et, étant la seule minorité nationale du pays, ils ont représenté une vitrine du régime.

Rappelons que le territoire où sont installés les Sorabes comprend dans le sud-est du Land de Saxe la Haute-Lusace: adossée au sud aux Monts de Lusace formant la frontière avec la République tchèque, bordée à l'est par la Neiße formant frontière avec la Pologne, elle atteint les villes de Bischofswerda et de Kamenz à l'est de Dresde. C'est là qu'habitent les Haut-Sorabes qui parlent le haut-sorabe, assez proche du tchèque. $\mathrm{Au}$ nord de ce territoire s'étend en continu au Brandebourg la Basse-Lusace, longeant la frontière Oder-Neiße, remontant vers le nord jusqu'à une centaine de kilomètres de Berlin, à l'ouest vers Luckau et Finsterwalde. Elle est peuplée par les Bas-Sorabes parlant le bas-sorabe, plus proche du polonais. La population sorabe ne dépasse pas aujourd'hui 60 à 65000 personnes.

La première formulation de mon sujet était: "Comment les Sorabes ont-ils perçu la RDA ?», sujet qui aurait supposé une vaste enquête sociologique, longue et difficile dans un État où il n'existait pratiquement pas d'opinion publique, et où les citoyens se faisaient discrets par prudence. Dans une première approche, il m'a semblé plus commode d'interroger les écrivains par le biais de leurs écrits. Je vais donc chercher à voir comment les écrivains sorabes se sont exprimés sur le sujet. Il s'agit des écrivains les plus féconds, les plus publiés et les plus lus en Lusace, donc à ce titre les plus représentatifs; nous citerons les noms de Jurij Brězan (1916-2006), Jurij Koch (1936), Mĕrćin Nowak-Njechorński (1900-1990), Jurij Krawža (1934-1995), Angela Stachowa (1948), Křesćan Krawc (1938).

* Chargé de cours complémentaire à l'Institut national des langues et civilisations orientales (Inalco). 
Ce que les écrivains ont perçu se rapporte aux différents aspects de la RDA: non seulement le régime, les institutions et la vie politique, mais aussi et surtout le reflet de ces institutions sur la vie quotidienne et sur les mentalités. Les choses se sont grosso modo passées en trois temps:

1. Le temps de l'approbation / de l'adhésion: de 1949 aux années 1950-1960

2. La critique masquée: des années 1960 aux années 1990

3. Le regard rétrospectif, postérieur à la chute du Mur.

Signalons que tous les textes cités ont été traduits par l'auteur de cet article, et qu'il s'agit pour tous sauf un d'une première parution.

\section{Le temps de l'approbation / de l'adhésion}

Ce temps correspond à la mise en place d'un nouveau régime, qui a précédé la création de la RDA. Dès mars 1948 a été votée à la Diète de Saxe une loi de protection des Sorabes qui leur assure pour la première fois de leur histoire la reconnaissance de leur identité et le soutien financier de leurs activités culturelles. Leur organisation nationale, la Domowina, a été recréée dès mai 1945 et rapidement autorisée par l'Administration militaire soviétique. Après avoir hésité entre indépendance, rattachement à la Tchécoslovaquie et autonomie, la Domowina accepte en novembre 1947 la formule de l'autonomie culturelle au sein d'un futur État-SED. En échange de leur acceptation de rentrer dans le rang, c'est-à-dire de ne pas présenter de liste électorale séparée, mais de faire figurer leurs candidats sous l'étiquette SED, les Sorabes vont avoir droit à toute une série d'institutions financées par cet État: enseignement officiel du sorabe, institut de formation des maîtres, section de sorabistique à l'Université de Leipzig, institut de recherche, maison d'édition, musée d'art populaire, studio de cinéma, ensemble folklorique, théâtre germano-sorabe, revues pédagogiques et scientifiques, quotidien et hebdomadaire, journaux pour enfants, théâtre de marionnettes, etc. C'est là pour les Sorabes une grande satisfaction et cela se ressent dans les publications des écrivains.

Le premier à publier après la Seconde Guerre mondiale est Jurij Brězan; c'est le nom le plus important de la littérature sorabe contemporaine. Il a été l'auteur le plus productif: on compte une bonne soixantaine d'ouvrages à son actif, traduits en une vingtaine de langues, sans parler des livres pour enfants; c'est lui qui a vécu le plus longtemps, jusqu'à 90 ans - il est mort en 2006 -, et il a été le plus honoré de son vivant: vice-président de l'Union des écrivains de RDA, membre du Pen-club, de l'Académie des Arts berlinoise et titulaire de nombreux prix littéraires et distinctions diverses. Il avait même occupé de hautes fonctions à la Domowina et dirigé la brigade de Jeunesse Sorabe, qui avait participé à la construction d'une ligne de chemin de fer en Yougoslavie en 1947. Il sera membre du Parti SED de 1946 à 1990.

Le premier petit roman qu'il publie en 1949, Madelena, est une sorte d'idylle paysanne; le narrateur est un agriculteur heureux qui a trouvé la femme de sa vie et fait avec elle le bien autour d'eux. Ils font tous deux partie de nombreuses associations locales, sont les premiers à remettre les quotas de lait et de grains exigés par les autorités et font de leur village un village-modèle, ce qui vaut à ce dernier moult récompenses. L'adaptation aux conditions du nouveau régime est donc parfaite ${ }^{(1)}$.

1 Jurij BRĚZAn, Zhromadźene Spisy I, Budyšin, Ludowe nakładnistwo Domowina, 1965, p. 35-136. 
La même année, Brězan commence un roman dont il publiera les fragments dans le quotidien Nowa Doba et, 16 ans plus tard, dans le volume où il rassemble ses écrits de jeunesse ${ }^{(2)}$. On y voit un soldat rentrer de captivité après dix ans d'absence. Au cours de ses pérégrinations, il a découvert d'autres horizons et estime qu'il ne peut plus cultiver son bout de terre en s'échinant du matin au soir comme son père avec des moyens primitifs. Épaulé par le secrétaire de la section locale du SED, il va concevoir un centre de machines agricoles au service de la collectivité, après avoir convaincu les paysans du village qu'il ne leur propose pas un kolkhoze. C'est, avant l'heure, un héros socialiste que l'écrivain met ici en scène.

En 1950, c'est à la revue culturelle sorabe Rozhlad qu'il donne une nouvelle intitulée Nowa metoda (La nouvelle méthode) ${ }^{(3)}$, où un forestier finit par comprendre qu'il faut sortir des anciennes habitudes, innover pour dépasser les normes, car «les normes, non, ce n'était pas si méchant que ça ${ }^{(4)}$.

En 1951, l'auteur reprend la question des stations collectives de machines agricoles qui font si peur aux petits exploitants, lesquels refusent l'idée de kolkhozes. Auf dem Rain wächst Korn (Des céréales poussent sur le bord du champ) est le premier écrit publié en allemand sur le conseil de Ludwig Renn ${ }^{(5)}$. On y voit un paysan qui comprend qu'il ne pourra jamais labourer tout seul avec ses deux vaches aussi vite que le tracteur de la station agricole et qu'il se ruinera la santé à vouloir rivaliser avec les nouvelles machines. Il finit par céder et non seulement fait labourer et semer son champ par les machines de la station, mais va jusqu'à supprimer le bornage avec le voisin pour gagner de la terre cultivable, ouvrant ainsi le chemin à la collectivisation des terres.

Dans une autre nouvelle du recueil Tučny wołojnik (Le crayon gras) ${ }^{(6)}$, l'engagement responsable de deux agriculteurs et l'intervention d'un député à la Chambre du Peuple permettent de mettre fin au tripatouillage des chiffres pratiqué par les scribes de la laiterie: force restera au droit - les institutions fonctionnent.

La nouvelle la plus connue et la plus traduite est Stara Jančowa (La vieille Jančowa) ${ }^{(7)}$. C'est l'histoire d'une simple paysanne pauvre qui a affronté toute sa vie "les autorités » - que ce soit le métayer qui l'emploie, la commune, le policier ou les nazis - et ne verra son sort s'améliorer qu'au lendemain de la Seconde Guerre avec le nouveau régime, qui augmente sa pension et lui redonne les droits précédemment supprimés. Elle devient sous la plume de Brězan la figure emblématique de la prolétaire des campagnes qui prend conscience du fait que les choses ont changé:

«- Les autorités? La grand-mère réfléchit - Oui, les autorités, avant c'étaient les autres et aujourd'hui - aujourd'hui - c'est nous.

- Qui ça, nous?

2 Ibid., Přewrót, p. 155-203.

3 Nowa metoda, Rozhlad, 09.05.1950, p. 27-36.

4 Ibid., p. 29: "Z nórmami - to docyła tak zlóstniske njeběše ».

5 Auf dem Rain wächst Korn, in: Sorbische Erzählungen und Gedichte (Nouvelles et poésies sorabes), Berlin, Verlag Volk und Welt, 1951; le texte sorabe: Na mjezy rosće žito (même titre) devait paraître l'année suivante dans Stara Jančowa, Dwě nowelce (La vieille Jančowa, Deux petites nouvelles) p. 56-94 et être repris en 1965 dans les Zhromadźene spisy I (CEuvres complètes), p. 310-345.

6 Tučny wołojnik, in: Zhromadźene spisy I (note 1), p. 259-275.

7 Stara Jančowa, Dwě nowelce (note 5), p. 56. 
- Eh bien, la famille Duban, l'oncle et leur tante, et le Président, et Wićaz et...

- Et toi aussi, grand-mère?

- Oui, moi aussi, affirma la vieille Janowa. Puis elle hocha la tête deux ou trois fois et confirma:

- Maintenant je suis moi aussi les autorités.

Elle eut un petit sourire:

- Je veux dire, fit-elle, un tout petit bout des autorités " ${ }^{(8)}$.

On a noté qu'il est question du Président; il s'agit du premier Président de la RDA, Wilhelm Pieck (1876-1960), figure populaire chez les Sorabes grâce à son abord facile et ses manières directes. Brězan l'évoque dans une autre nouvelle publiée en 1952 dans la revue Rozhlad ${ }^{(9)}$. Le Président fait un discours à Budyšin/Bautzen et, au cours d'un bain de foule, accepte d'un paysan sorabe un épi qu'il met dans sa poche; son comportement favorable aux Sorabes amène un autre paysan à livrer un cochon de plus que prévu par les quotas officiels.

Dans un recueil de poèmes datant de 1955, l'écrivain lui dédie Prezidentej zbožo (Du bonheur au Président) ${ }^{(10)}$ dont la dernière strophe souhaite:

«Lěpše njewěm Tebi přeć «Je ne peux te souhaiter mieux

hač

zbožo

que

za nas wšěch!»

du bonheur

pour nous tous!»

On a la même évocation de «Naš prezident» (Notre Président) dans le roman Jan. Roman pytaceho čłowjeka (Jan, roman d'un homme qui cherche) de Kurt Krjeńc, dont Rozhlad présente un extrait p. 209-213.

Alors qu'il travaille à un roman et se cherche un héros, Brězan écrit encore en 1963 dans un article intitulé "Mehr als die Taten des Herakles» (Plus que les travaux d'Hercule)» :

«Mon roman essaie d'englober la période 1946-1962, je ne réussirai pas tout à fait à donner forme à ce que j'ai compris: une des réalisations les plus grandioses de notre temps est la réalisation des millions d'hommes simples du quotidien, qui d'un bout d'Allemagne de 1945 ont créé la RDA de 1963 ! " ${ }^{(11)}$

8 Zhromadźene spisy I (Euvres complètes), p. 394:

" "Wyšnosć?" premyslowaše stara. "Haj, wyšnosć: prjedy su to byli druzy - a dźensa - dźensa smy to my."

"Štóha - my?"

"Nó, Dubanec wuj a jich ćeta a - a prezident - a Wićaz - a..."

"A ty tež wowka?"

"Haj, ja tež”, twjerdeše stara Jančowa. Potom kiwny dwójce, trójce z hłowu a potwjerdźi: "Nětko sym tež ja wyšnosć.” Zasmja so trochu: "Měnju”, přispomni, “mały kusćićk wyšnosċe”."

9 Rozhlad, janvier-février 1952, p. 4, 9, 10, extrait de 52 Wochen sind ein Jahr (52 semaines font une année).

10 Prezidentej zbožo, Naš wšědny dźeń (Notre quotidien), Budyšin, Ludowe nakładnistwo Domowina, 1955 , p. 11.

11 «Mehr als die Taten des Herakles», in: Ansichten und Einsichten (Vues et points de vue), Berlin, Verlag Neues Leben, 1976, p. 50: «Mein Roman versucht, die Zeit von 1946-1962 einzufangen, es wird mir nicht voll gelingen, das nachzugestalten, was ich begriffen habe: Eine der großartigsten Leistungen unserer Zeit ist die Leistung der Millionen einfacher, täglicher Menschen, die aus einem Stück Deutschland von 1945 die DDR von 1963 geschaffen haben!» 
En 1955, le peintre-écrivain Měrćin Nowak-Njechorński rappelle dans Rozhlad ${ }^{(12)}$ la création des premières coopératives agricoles et d'élevage que tous finissent par accepter. Il commente: "Eh oui, c'étaient des rêves d'avenir. Mais nous vivons une époque où les rêves les plus hardis deviennent réalité!» ${ }^{(13)}$ Et Měrćin Nowak termine ainsi son article:

«Les coopérateurs ont commencé à améliorer leur existence, ont commencé à changer et aménager le village, en ont fait le centre et la bastille d'une vie nouvelle, un maillon de la vie d'un village progressiste: une colonie socialiste à Lěšćiny» ${ }^{(14)}$.

Coopératives, progressisme et socialisme sont étroitement liés, c’est la théorie officielle. Měrćin Nowak-Njechorński restera jusqu'au bout un militant sincère et un inconditionnel de la RDA; il mourra en 1990, de la fin de la RDA, pourrait-on dire.

Jurij Brězan fait le point en 1976 et constate que «la littérature a, au cours des trois dernières décennies de ce millénaire, rempli sa tâche», et il ajoute:

«Dans ce but, nous sommes là, nous les écrivains qui, dans les vingt dernières années, avons aidé cette République à devenir notre République dans des millions de cerveaux. Des écrivains qui sont aussi fiers - et ce n'est pas notre moindre fierté - d'être restés sans s'égarer tout au long de ces vingt années derrière le Parti de la classe ouvrière et leur Comité central. Et il en sera de même dans l'avenir. Cela ne saurait faire débat » ${ }^{(15)}$.

Il ne faut pas s'étonner de ce concert d'éloges du régime dans un premier temps. La population sorabe a connu l'asservissement durant un millénaire et la soumission aux grands propriétaires terriens, dont elle se voit enfin définitivement libérée. De plus, elle vient de subir douze ans de nazisme destructeur de sa langue et de son identité; le nouveau régime signifie donc pour elle une renaissance non seulement culturelle, mais aussi sociale et économique - dans un premier temps, tous les espoirs sont permis.

\section{La critique masquée}

Les déceptions vont néanmoins se manifester peu à peu, une fois la première vague d'enthousiasme passée. L'évolution du régime va confronter les Sorabes à des réalités nouvelles qui ne sont pas toutes réjouissantes. C'est dans l'espace réduit de la liberté d'expression qu'il faut chercher désormais l'écho de ces perceptions chez les écrivains. L'image de la RDA va y apparaître en creux plutôt qu'en relief et on évolue dans le domaine de la référence et de l'allusion, voire de la parabole. Comme dans tous les

12 «Lěšćiny», Rozhlad, 1955, p. 257-265.

13 Ibid., p. 259: "Nó, to běchu sonjenja wo přichodźe. Ale smy žiwi w dobje, hdyž tež najchrobliše sonjenja so přestworjeja do woprawdźitosće!»

14 Ibid., p. 265: «Prodruswownicy su započeli wjes přeměnjeć a sporjedźić, woni su załožili srjedźišćo a twjerdźiznu noweho lěpšeho žiwjenja, wjazadło noweho, doprědkarskeho wjesnanstwa: socialistiske sydlišćo na Lěšćinach.»

15 Von Enge und Weite (Étroitesse et largeur de vues), in: Ansichten und Einsichten (note 11), p. 126: «festgestellt werden kann, daß die Literatur in den letzten drei Jahrzehnten dieses Jahrtausends ihre Aufgabe erfüllt habe... Zu diesem Zwecke also sitzen wir hier, wir Schriftsteller, die in den vergangenen zwanzig Jahren dieser Republik geholfen haben, in Millionen Hirnen zu unserer Republik zu werden. Schriftsteller, die auch darauf stolz sind - und nicht zuletzt darauf - unbeirrt in allen Situationen dieser zwanzig Jahre hinter der Partei der Arbeiterklasse und ihrem Zentralkomitee gestanden zu haben. Daran wird sich auch in Zukunft nichts ändern. Das steht hier überhaupt nicht zur Debatte.» 
pays du socialisme dit réel, l'expression littéraire est à double fond et le lecteur a pris l'habitude de sonder l'écrit pour comprendre le message. D'une certaine manière, tout est dit, mais sous le masque, qu'il faut lever pour percevoir l'intention de l'écrivain. Le lecteur est devenu peu à peu un décrypteur. Il reste néanmoins un espace de critique acceptée comme positive; mais cette critique vise les hommes à différents niveaux, sans que le Parti soit nommément concerné.

À partir des années 1960 se développe en Lusace une campagne systématique de regroupement des terres en coopératives agricoles: on veut amener les paysans à donner leurs terres et leur bétail à la coopérative. C'est en principe un acte volontaire, mais en réalité on fait pression sur eux et les activistes du Parti rendent aux récalcitrants d'incessantes visites. Dans un article publié en 1976, "Wissen und Macht», Jurij Brězan rappelle le cas d'un paysan septuagénaire qui accepte de céder sa vache et son champ à la coopérative, mais qui se sent trop vieux pour y adhérer lui-même:

«Durant deux nuits, l'agitateur proclama dans un haut-parleur: "personne n'est trop vieux pour le socialisme!" Quelqu'un sauva alors le vieux et sa femme des projecteurs et des haut-parleurs » ${ }^{(16)}$.

Les paysans s'accrochent à des terres héritées de leurs pères, appartenant à leur famille depuis des décennies, ils veulent "travailler avec goût et amour», mais ne veulent pas «travailler par équipes». Ils veulent rester dans leurs fermes entourés de leurs "enfants, petits-enfants, brus et gendres " ${ }^{(17)}$, et leur résistance s'éternise. Tous les écrivains sorabes s'en font l'écho. Le passage à la LPG (Landwirtschaftliche Produktionsgenossenschaft - coopérative agricole de production) est souvent plus théorique qu'effectif. Křesćan Krawc note dans un récit romancé:

«Il y avait le journal sous la porte, avec sous nos yeux un gros titre comme je n'en avais encore vu dans aucun journal: "Der Sozialismus hat gesiegt, auch auf dem Lande. Vom Ich zum Wir - alle Bauern in der LPG!” " ${ }^{(18)}$.

Le passage à l'allemand dans un texte sorabe souligne bien l'origine officielle du slogan descendu d'en haut. La décision des paysans a été néanmoins purement formelle, même si on a élu comme président de la LPG un des militants qui ont fait du forcing - en fait, chacun continue à cultiver son champ...

Le plus sensibilisé à ce problème est justement Křesćan Krawc, journaliste écrivain, qui relate la vie d'une coopérative agricole en 1973 dans son ouvrage $W$ delanach na Katyrnu (Dans le Plat-pays à la Sainte Catherine, 1982). On a ici affaire à une littérature qui s'abrite derrière le reportage.

16 «Wissen und Macht» (Savoir et Pouvoir), in: Ansichten und Einsichten (note 11), p. 65: «Zwei Nächte lang erklärte dann der Agitator durch den Lautsprecher: 'Niemand ist zu alt für den Sozialismus!' Irgend jemand rettete damals den Alten und seine Frau vor Scheinwerfern und Lautsprechern. »

17 Křesćan Krawc, W delanach na Katyrnu (Dans le Plat-pays à la Sainte Catherine), Budyšin, Ludowe nakładnistwo Domowina, 1979, p. 102: «Wón chce dźěłać z lóštom a lubosću, ale nochce w změnach dźěłać... Jeho dla bychu móhli wšitcy doma wostać: dźěći, wnučki, přichodne dźowki a přichodni synojo.»

18 Křesćan KrawC, Wočakńmy nalěćo (Attendons le printemps), Budyšin, Ludowe nakładnistwo Domowina, 1989, p. 374: «Pod durjemi tčeše nowina, před našimi wočemi nadpismo, tak wulke, kajkež hišće w žanej nowinje widźał njeběch. " (Le socialisme a vaincu. Même à la campagne. On passe du moi au nous - tous les paysans dans la coopérative!) 
Refusant l'embrigadement, certains agriculteurs veulent rester libres. L'auteur rappelle quand même que la vie du paysan libre "n'était pas comme dans un conte, la réalité était sans pitié. La liberté pour le paysan, c'était de s'échiner, de s'abîmer le dos, d'aller tard au lit et de se lever à quatre heures du matin ${ }^{(19)}$. Il consacre plusieurs pages $^{(20)}$ à rappeler les avantages des regroupements: accès aux machines agricoles des stations collectives, maîtrise des horaires de travail, assurance d'un salaire décent, protection et limitation du travail des femmes soumises à la double journée de travail, meilleure utilisation des animaux - les vaches ne s'épuisent plus à tirer la charrue et peuvent donner du lait. Mais il y a dans cette coopérative sorabe un dégât collatéral, qui redonne des arguments aux adversaires de la collectivisation. Une orientation productiviste, encouragée par la propagande gouvernementale, amène les coopératives à acheter toujours plus de bétail, sans toujours se soucier de l'achat de fourrage et de l'hébergement des animaux. C'est ainsi qu'un troupeau de 100 génisses attend dans la première neige l'abri promis depuis longtemps, une étable dont la construction tarde. L'auteur n'utilise pas les guillemets, car le président de la coopérative se fait la vox populi, ses propos reflètent l'opinion générale - et sans doute aussi celle de l'auteur:

«Dans un premier temps, c'était l'urgence - ceux de là-haut avec leurs grands slogans et encore et toujours: des millions, des millions pour le bétail, pour le combinat - et maintenant on ne voit plus personne. Les millions sont dans la boue. Le bétail est là, mais l'étable n'est pas encore prête! » ${ }^{(21)}$

Le groupement de coopératives organise une réunion comportant un ordre du jour formaliste et très strict, qui ne permet pas au responsable de la coopérative concernée de se faire entendre et de signaler le danger couru par les animaux bloqués dans la neige.

Le président de la coopérative se pose la question:

«Comment est-ce possible - dans une société organisée, quinze ans après la collectivisation, une telle confusion, des situations extravagantes qui rappellent plus les lendemains de la guerre qu'une transition vers une agriculture industrielle. Est-ce que c'est normal?

Non, ce n'est pas normal, mais avec le temps, on s'est habitué à ces situations extravagantes. Et ce n'est pas bien de s'habituer à cela. Les projets, les bilans, les rapports - c'est surtout à cause de cela qu'on a changé de projets, modifié les bilans et parfois même écrit dans les rapports des choses contraires à la vérité» ${ }^{(22)}$.

Il précise un peu plus loin:

19 K. KRAwC, W delanach na Katyrnu (note 17), p. 83: «Bajkojte tole žiwjenje njebě, realita bě njesmilna. Swobodu za bura, zo by so dračował, sej křiž poškodźił, pozdźe do łoža chodźił a rano w štyrjoch stawał.»

20 Ibid., p. 56-60.

21 Ibid., p. 7: «Najprjedy su měli nuznje - ći wot horjeka, z wulkimi słowami a přeco zaso: Miliony, miliony za skót, za kombinat -, a nětko so nichtó wjace widźeć njeda. Nětk miliony w blóće leža. Skót tu je, ale hródź njeje hišće hotowa!»

22 Ibid., p. 121 : «Kak je to móžno - wosrjedź rjadowaneje towaršnosće pjatnaće lět po zdrustwowjenju, tajke šmjatańcy, ekstremne situacije, kotrež skerje na powójnski čas dopominaja hač na přechod $\mathrm{k}$ industrijowemu ratarstwu. Je da to normalne? / Normalne to njeje, ale počasu so na nje zwučimy, na tele ekstremne situacije. A njeje derje, zo so na to zwučimy. Plany, bilancy, rozprawnistwo zwětša tohodla, zo bychmy plany přemjetali, bilancy zranili a do rozprawow druhdy tež njewěrnosće napisali.» 
«Tant que nous entendrons dire à la radio qu'une fois de plus nous avons engrangé de bonnes récoltes, alors qu'en réalité nous sommes dans la boue jusqu'aux essieux, nous serons bien obligés de nous mentir entre nous. Et la réalité se venge cruellement à la fin de l'année en produisant des chiffres en rouge» ${ }^{(23)}$.

Et il reprend:

"On a par exemple commandé une machine, on a un contrat pour ça, et en pensée on travaille déjà avec. Et d'un coup arrive une lettre: en raison d'une décision du ministère machin et de la commission de district pour ça et ça, il n'est pas possible d'attribuer la machine. Et te voilà les mains vides dans ton champ. Personne ne t'a rien dit, ni rien annoncé, tu n'as qu'à t'incliner. Nous sommes aujourd'hui patients là où il ne faudrait pas l'être. Et nous sommes parfois impatients, là où il faudrait avoir de la patience » ${ }^{(24)}$.

Le financement a été en fait étalé sur une trop longue période et les tranches étaient trop réduites. Inconséquences dans la gestion... Et le président d'ajouter :

«Nous à la coopérative Sorabija, nous ne nous sommes pas fâchés comme il aurait fallu. Se fâcher, ça a aussi des inconvénients! Nous avons fait bonne figure, et ensuite, quand nous avons pété les plombs, nous avons donné du poing sur la table et dit qu'ils n'avaient pas le droit de penser, à Kamenz ${ }^{(25)}$, qu'on peut faire ce qu'on veut avec ces idiots de Sorabes » ${ }^{(26)}$.

Ici se fait jour un autre élément: l'administration allemande fait manifestement peu de cas des Sorabes et a sans doute redistribué les crédits. On frôle la discrimination. Il va de soi que les écrivains sorabes sont très sensibles à cet aspect.

Jurij Koch (né en 1936) illustre dans Mjez sydom mostami (Entre les sept ponts) la résistance de ces paysans qui se redistribuent les terres ne pouvant être traitées par les machines. Le roman nous montre un maire de village aux prises avec un président de coopérative manipulateur qui dresse le village contre lui. En dépit de son honnêteté, de sa rigueur et de ses bonnes idées, le maire prête le flanc par sa relation avec une femme mariée ou plutôt mal mariée et le Parti le fera partir: dans le village socialiste, il n'y a pas d'hommes nouveaux ${ }^{(27)}$.

Jurij Koch reprend les mêmes personnages dans Róžamarja (Rosemarie), qui est la suite de Mjez sydom mostami. Il parsème son récit de brèves remarques qui sont autant d'allusions souvent transparentes. Ainsi sur le rôle omniprésent du Parti, même en ce qui concerne l'activité d'une chorale; peut-être faudrait-il enrichir le répertoire par

23 Ibid., p. 123: "Tak dołho, kaž budźemy z radija słyšeć: Zaso dobre žně derje dochowali - ale we woprawdźitosći stejimy hač k wóskam w błóće, to so mjez sobu k łžam nuzujemy. Realita so surowje wjeći, na kóncu lěta z čerwjenymi ličbami.»

24 Ibid., p. 122: «To maš na přikład mašinu skazanu, maš za nju zrěčenje, w myslach hižo z njej dźěłaš. A naraz ći přileći pismo: Na zakładźe wobzamknjenja ministerstwa takatak a wobwodneje komisije za toato njeje móžno, mašinu dodać. - A stejiš z prózdnimaj rukomaj na polu. Nichtó ći njeje ničo rjekł, ničo připowědźił, maš so kłonić. My smy dźensa sćerpliwi tam, hdźež to njebychmy směli być. A smy druhdy njesćerpliwi, hdźež dyrbjeli sćerpnosć měć.» Chef-lieu de canton, dont dépendent les villages alentour.

Ibid., p. 122: «My w Sorabiji njejsmy so tak hóršili, kaž by trjeba było. Z tym hóršenjom- to ma swoje špaki! Smy přećelne mjezwoča pokazowali, a potom, hdyž je so nam tola kornar puknył, smy z pjasću wo blido dyrili a prajili, zo njesmědźa sej w Kamjencu myslić, zo móžeja z tymi hłupimi Serbami činić, štož chcedźa.» 
des chants évoquant le combat du prolétariat et ne pas se contenter de refrains comme duajdu, tralala ${ }^{(28)}$ ? C'est ce que se demande le secrétaire de la section locale du Parti, qui est par ailleurs un homme sympathique au courant des moindres détails de la vie du village (p. 92-99).

Lorsque le secrétariat de la župa ${ }^{(29)}$ s'installe dans les locaux d'un ancien salon de coiffure, on organise une fête et c'est l'occasion de discours officiels convenus et grandiloquents, louant entre autres la politique des minorités, assortie d'une citation ${ }^{(30)}$ :

«[...] car les minorités nationales offensées, il n’y a rien à quoi elles sont plus sensibles que l'égalité et le non-respect de cette égalité, que ce soit par négligence ou par plaisanterie, et le non-respect de cette égalité de la part de leurs camarades prolétaires... Lénine.»

L'enseignante qui est l'héroïne du roman disait juste avant la fête ce qu'elle en pensait:

«C'est comme ça... Du côté de la loi, tout est clair et on la cite très bien depuis déjà un quart de siècle dans tous les rapports. C'est l'amitié! Voilà le mot favori de toutes les tribunes et de toutes les places publiques! Et c'est en allemand qu'il sonne le mieux» ${ }^{(31)}$.

On aura noté l'ironie au passage.

«Les journaux vantent les coutumes populaires, mais font le silence sur le caractère religieux de certaines d'entre elles, comme les Cavaliers de Pâques, qui s'avancent précédés de leurs curés tenant d'énormes crucifix... On préfère parler des œufs peints » ${ }^{(32)}$.

L'auteur revient plusieurs fois sur une situation qui lui tient à cœur et relie le problème national à l'écologie: la destruction de la nature due à l'exploitation de la mine de lignite.

«Au bord de la fosse d'extraction se trouvait un banc. Les dents des tractopelles lui avaient fichu la paix; il était maintenant le signe d'une vérité de l'agit-prop: on ne mord pas sur le parc, votez pour les candidats du Front national ${ }^{(33)}$ ! Mais chacun voyait que personne ne pouvait plus s'asseoir là et chacun savait que le parc disparaîtrait de lui-même en raison de l'assèchement de la nappe phréatique " ${ }^{(34)}$.

Slogan politique et simple réalité sont deux choses différentes. Et l'héroïne se souvient d'un camarade de classe dont la maison a disparu depuis, avalée par la mine, tout comme la vieille église en bois du XIII ${ }^{\mathrm{e}}$ siècle qui se dressait à côté( ${ }^{(35)}$.

28 Jurij Koch, Róžamarja, Budyšin, Ludowe nakładnistwo Domowina, 1975, p. 116.

$29 Z \check{Z}$ pa: section régionale de la Domowina.

30 J. Косн, Róžamarja (note 28), p. 104: «[...] weil die gekränkten nationalen Minderheiten für nichts ein so feines Gefühl haben wie für die Gleichheit und für die Verletzung dieser Gleichheit, sei es auch aus Fahrlässigkeit, sei es auch nur im Scherz, für die Verletzung dieser Gleichheit durch ihre Genossen Proletarier... Lenin.»

31 Ibid., p. 103: «Tak to je... Zakonsce je wšo jasne a da so derje citować, štwórć lětstotka dołho hižo we wšěch referatach. Přećelstwo! Kajke to woblubowane słowo na wšěch tribunach a naměstach. A němsce klinči to najlěpje.»

32 Ibid., p. 65.

33 «Nationale Front»: liste d'union regroupant les différents partis autorisés.

34 Ibid., p. 134: «Na kromje twarskeje jamy steješe ławka. Ju běchu zuby bagrow na pokoj wostajili: Nětko bě wona znamjo za agitacisku wěrnosć: Park so njenakusa, wolće kandidatow Nacionalneje fronty! Ale kóždy widźeše, zo tu nichtó wjace sydać njemóžeše, a kóždy widźeše, zo so park sam wot so zhubi, dla ćeknjeneje zemskeje wody.» 
La destruction de l'environnement, de l'air en particulier, par l'industrie extractive est évoquée par Jurij Koch dans tous ses livres, entre autres dans Le Cerisier (Der Kirschbaum en allemand, Wišnina en sorabe), dans lequel le grand-père sectionne la couronne de son arbre et la plonge symboliquement dans l'étang pour la régénérer ${ }^{(36)}$.

Dans une autre nouvelle, Landvermesser (Géomètres), l'auteur souligne la désinvolture de géomètres venus piqueter le parcours d'une future route devant mener à la mine et passer sur la maison où ils sont reçus. Excavateurs, tractopelles, bruit d'enfer et fumées toxiques crachées par des machines qui vont chasser les hommes et faire disparaître une vie tissée de traditions et marquée d'un bonheur simple ${ }^{(37)}$.

Dans un poème qui eut un grand retentissement, le poète Kito Lorenc exhale en 1966 toute sa nostalgie du pays où il a vécu ses premières années enchantées par les beautés de la nature. Et il pleure sur «la belle Lusace, paradis souillé», «joyau de mon enfance qui moisit dans la boue» ${ }^{(38)}$. Il fut l'auteur d'un scénario de film sur le même sujet qui porte le même titre.

Angela Stachowa (née en 1948) intitule un petit roman Vineta, du nom d'une ville mythique engloutie, métaphore d'un village abandonné laissé en pâture à la mine, qu'une jeune fille découvre au hasard d'une promenade en vélo ${ }^{(39)}$. Křesćan Krawc rencontre quant à lui une femme qui a été relogée après avoir dû abandonner sa ferme devant l'avance de la mine:

«Elle resserra le nœud de son fichu et fronça les sourcils en regardant les grands ensembles:

“Moi aussi, j'ai mon logement dans un ensemble comme ça, avec l'eau jusque dans les cabinets, mais je n'ai pas d'appentis. Quand on doit quitter sa maison, ce n'est pas bien! Rester là où on a grandi, voilà ce qui a de la valeur dans la vie. Il a fallu emballer ses frusques, décaniller et tout recommencer depuis le début" " ${ }^{(40)}$.

Jurij Koch aborde aussi un autre problème: celui du politiquement correct. Il le fait prudemment par le biais de l'école. Le conseil des professeurs du lycée doit statuer sur le certificat de maturité de fin d'études d'une élève d'après le mémoire qu'elle a remis. Mais celui-ci est en fait centré sur un dramatique problème familial, et le sousdirecteur de l'établissement, membre du Parti, n'accepte pas qu'un problème privé passe avant l'expression d'une conscience politique; il exige la réécriture du mémoire. D’où le silence général, où se fait jour la question de savoir si, écrit l'auteur, «d'une

36 Jurij Koсн, Der Kirschbaum, Halle/Leipzig, Mitteldeutscher Verlag, 1984, p. 44. Édition sorabe: Wišnina, Budyšin, Ludowe nakładnistwo Domowina, 1984, p. 28.

37 Jurij Косн, Landvermesser, in: Der einsame Nepomuk, Halle/Leipzig, Mitteldeutscher Verlag, 1978, p. 6-55. En sorabe: Kotjatkowa dźiwna lubosć, in: Wosamoćeny Nepomuk, Budyšin, Ludowe nakładnistwo Domowina, 1978, p. 7-43.

38 Kito Lorenc, SP Serbska poezija, Budyšin, Ludowe nakładnistwo Domowina, 1979, "Struga», p. 47-49: «Domizna, rjana Łužica, mazany raj» / «dźěčatswa kamušk, plěsniwiš nam w błóće», p. 48.

39 Angela Stachowa, Vineta, Budyšin, Ludowe nakładnistwo Domowina, 1983, p. 16-17.

40 K. KRAwC, Wočakńmy nalěćo (note 18), p. 382 : «Wona sej suk při rubišku krućišo zwjaza a při tym z pochmurjenym čołom k blokomaj pohladny. "Mam tež swoje bydło w tajkim bloku, wodu hač na hajzl, ale žanu kólnju. Hdyž dyrbi čłowjek wot doma preč - to ničo rjane njeje! Wostać, hdźež sy wotrostł, to je wjele hódne w žiwjenju. My dyrbjachmy naše zmachi spakować a wotsal ćahnyć a zaso wotprědka započeć.” " 
manière non scientifique et contrairement à notre point de vue, lorsqu'il s'agit d'une vérité privée, la somme des vérités privées ne constitue pas une partie de la vérité de la société » ${ }^{(41)}$. Le župan de la section locale de la Domowina intervient et cite le cas d'un journaliste ${ }^{(42)}$ qui a perdu son poste pour avoir refusé de modifier un article devant illustrer une campagne condamnant «les tendances petites-bourgeoises» au détriment de la mémoire de son grand-père, Arnošt Bart, militant courageux de la cause sorabe ${ }^{(43)}$. Et il précise: «Un socialiste dit honnêtement et ouvertement son opinion. Je ne suis pas enseignant, mais pour cette partie de son mémoire, l'élève Liza mérite la note $1{ }^{(44)}$. Finalement les choses s'arrangeront grâce à l'intervention d'un parent d'élève qui est un ouvrier carrier et rappelle au sous-directeur que l'avenir de la jeune fille est en jeu.

La trilogie de Jurij Brězan autour du personnage de Felix Hanuš est un Bildungsroman, dont le troisième volume se situe à l'époque de la RDA; le titre sorabe est Zrałe lěta (Les Années de maturité), la version allemande s'appelle Mannesjahre (L'Âge d'homme) ${ }^{(45)}$. Le héros est un garçon sincère qui finit par adhérer au Parti et deviendra même secrétaire de section et président de la coopérative. C'est donc un citoyen qui joue les cartes de la société dans laquelle il vit. Il organise dans le manoir de l'ancien hobereau passé à l'Ouest un centre de formation pour adultes, où l'on enseigne entre autres le sorabe. Mais on décide en haut lieu de transformer ce centre en une «Maison de la culture cantonale pour la jeunesse des campagnes» ${ }^{(46)}$. On verra par la suite la section cantonale du Parti envoyer sur place un peintre officiel; celui-ci produira dans le style du réalisme socialiste toute une série de toiles "peignant en une semaine plus de vaches qu'il n'y en avait en tout dans les étables du village » ${ }^{(4)}$, toiles que le Parti lui achètera... car «il a aussi aidé le Conseil cantonal, qui a aussi bien apporté la culture à la campagne que donné la promotion à l'art selon le Plan» ${ }^{(48)}$. Notre héros fait face à un ancien camarade de classe devenu maire, un type d'homme qui contraste avec le héros Felix, un certain Rumbo au surnom caractéristique. Promu maire par les Soviétiques en 1945 grâce à un papier indiquant son appartenance au parti communiste d'avant-guerre, c'est un opportuniste brutal qui va faire carrière et s'opposera à Felix

41 K. Koch, Róžamarja (note 28), p. 181: «hač je njewědomostne a našemu stejišću njewotpowědne, hdyž wo priwatnu sprawnosć dźe, hač njewučini suma priwatnych sprawnosćow dźěl towaršnostneje wěrnosće."

42 Ibid., p. 185-186.

43 Arnošt Bart s'engagea au lendemain de la Première Guerre mondiale pour l'indépendance de la Lusace et fut condamné par un tribunal allemand à deux ans et demi de forteresse pour révélation de secrets militaires, qui contrevenaient en fait aux dispositions du Traité de Versailles.

44 C’est-à-dire la meilleure note. K. КосH, Róžamarja (note 28), p. 186: "Socialist praji sprawnje a wotewrjenje swoje měnjenje. Njejsym wučer, ale za tutón dźěl nastawka zasłuži sej Liza jedynku. »

45 Jurij BrĚZAn, Mannesjahre, Berlin, Verlag Neues Leben, 1964 / Zrale lěta, Budyšin, Ludowe nakładnistwo Domowina, 1964.

46 Ibid., p. 115: «Kreiskulturhaus der Landjugend».

47 Ibid., p. 186: «der Maler verfertigte in einer Woche mehr Kühe, als insgesamt in den Ställen des Dorfes standen ».

48 Ibid., p. 187: "Und geholfen war auch dem Kreisrat, der sowohl Kultur aufs Land gebracht als auch, dem Plan entsprechend, die Kunst gefördert hatte.» 
en maintes occasions. Un ami, communiste sincère en stage à Moscou, écrit à Felix, qui lui a fait part de sa déception: "Il y a des camarades qui croient qu'on doit traiter les camarades comme si les camarades n'étaient pas des êtres humains ${ }^{(49)}$. Notons que l'écrivain met habilement ce jugement sous la plume d'un communiste estimable. Ce propos sera illustré par l'intransigeance du maire, qui justifie une mesure impopulaire en disant: "C'est une décision qui vient d'en haut» ${ }^{(50)}$. Et le même Rumbo rappelle à Felix: «Moi, c'est le Parti qui m’a fait maire. Ce n'est pas moi, c'est le Parti que tu critiques ${ }^{(51)}$. Au cours d'une conversation avec son ami rentré de Moscou, Felix enfonce le clou:

"J'ai lu un livre, il y a dedans un homme du Parti qui sait tout, fait tout, n'a jamais de doutes, ne raconte pas de blagues idiotes comme toi. S'il y avait des gens comme cela, cinq suffiraient, un seul sur chaque continent, pour mener toute l'humanité tout droit et sans problème au paradis » ${ }^{(52)}$.

On aura noté un humour transparent pour qui vit en RDA. La section locale du Parti est convoquée pour décider de la suite à donner à l'accusation visant une paysanne mal embouchée que Rumbo taxe de contre-révolutionnaire, ce que personne ne croit au village. Mais la situation politique dans le monde actuel doit aussi être appréciée pour pouvoir juger du cas (p. 334). Et l'auteur se fait un malin plaisir de souligner l'absurdité et le ridicule des débats (p. 335-338). Il avait déjà révélé le fond de sa pensée en disant au fonctionnaire psychorigide qu'est le maire: «Un jour tu comprendras même, Rumbo, qu'il n'y a pas d'autre voie menant au socialisme que celle qui passe par la raison des hommes ${ }^{(53)}$.

Une occasion va mettre franchement l'écrivain de mauvaise humeur. En avril 1964 est promulguée une ordonnance modifiant la loi scolaire: désormais l'enseignement du sorabe sera soumis à la demande expresse des parents. Le sorabe devient en quelque sorte une affaire privée et l'on ne peut plus faire de publicité pour recruter. Le nombre des élèves apprenant le sorabe tombe alors de 12000 à 4000 . Jurij Koch montre dans Mjez sydom mostami comment un directeur d'école annonce la nouvelle disposition et fait pression sur les parents pour les amener à désinscrire leurs enfants du cours de sorabe ${ }^{(54)}$. Brězan avait composé en février 1950, dans l'euphorie de la visite en Lusace du Président Pieck, un poème Wie ich mein Vaterland fand (Comment j'ai trouvé ma patrie) ${ }^{(55)}$; l'indignation lui fait écrire dans la nuit qui suit la nouvelle disposition

49 Ibid., p. 122: «Es gibt Genossen, die glauben, mit Genossen müßten sie umgehen, als wären Genossen keine Menschen.»

50 Ibid., p. 147: "Das ist eine Anordnung von oben.»

51 Ibid., p. 148: «Mich hat die Partei zum Bürgermeister gemacht. Du kritisierst nicht mich, du kritisierst die Partei.»

52 Ibid., p. 211: «Ich habe ein Buch gelesen, darin lebt ein Mann von der Partei, der alles weiß, der tut alles, hat nie Zweifel, er macht auch keine dummen Witze, wie du - wenn es solche Menschen gäbe, fünf davon genügten, auf jedem Erdteil einer, um die ganze Menschheit geradeaus und ohne alle Umstände ins Paradies zu führen.»

53 Ibid., p. 283-284: «Einmal, Rumbo, wirst du sogar begreifen, daß es keinen Weg zum Sozialismus gibt außer die Vernunft der Menschen.»

54 Jurij Косн, Mjez sydom mostami, Budyšin, Ludowe nakładnistwo Domowina, 1968, p. 56-61 et 91-92. Swět budźe rieńši (Le monde sera plus beau), Bautzen, Institut für sorbische Volksforschung, 1951. 
officielle un autre poème: Wie ich mein Vaterland verlor (Comment j'ai perdu ma patrie). Et il porte le manuscrit au Politbüro du SED:

«Mein Sohn spielte im Sand malte ein $\mathrm{D}$ und ein $\mathrm{D}$ und ein $\mathrm{R}$ und meinte ein Vaterland.

Es wird das seine nicht sein und war das meine nicht mehr. Ich setzte den Fuß in den Sand und wischte es aus.»

\author{
«Mon fils jouait dans le sable \\ traçait un $\mathrm{D}$, un $\mathrm{D}$ et un $\mathrm{R}$ \\ désignant une patrie. \\ Ce ne sera pas la sienne \\ et ce n'était plus la mienne. \\ Je mis le pied dessus et effaçai \\ les lettres dans le sable ${ }^{56}$.
}

Le texte ne fut finalement imprimé qu’en 1993. La version que Brězan donne de l'événement est par ailleurs contestée par Jurij Grós dans un livre publié après la mort de l'écrivain ${ }^{(57)}$, ainsi que par l'historien Timo Meškank ${ }^{(58)}$. Il est permis de penser qu'une riche imagination joue des tours à la mémoire d'un écrivain... Toujours est-il qu'il fallut attendre 1968 pour que soit rétabli le droit de faire de la publicité pour l'enseignement en sorabe. Dans le livre consacré à la mort du "vieux père», Stary nan (traduit par Bild des Vaters en allemand et L'image du père en français), l'auteur fait allusion à un autre problème et fait dire au grand-père:

«Le socialisme n'est pas mauvais, un peu trop bruyant peut-être, je veux dire dans le journal ou à la télé, et aussi parce qu'il veut toujours avoir l'air d'un gâteau, quand le pain est aussi quelque chose de très bon. Mais ce qui ne me plaît pas dans ce socialisme, c'est qu'on vole tellement ... Par exemple ce Pětr de la colonie, il transporte le sable pour le combinat, il vide chez lui une remorque, il n'a pas besoin de sable, sa maison est terminée, le garage aussi. Comme il transporte du sable, il vole du sable. Tout le monde le sait, mais personne ne dit rien. Parce que tout le monde vole» ${ }^{(59)}$.

Jurij Krawža (1934-1995) dans Kupa moja starosć (Mon lot, c'est mon affaire) ${ }^{(60)}$ met en scène un paysan têtu qui rejoue le meunier de Sans-Souci, résiste à toutes les pressions psychologiques et refuse obstinément de céder son jardin enclavé dans un champ collectif. Dans Hany Mudrineje hotel (L'hôtel de Hana Mudrina, 1976) une paysanne retraitée rêve de transformer sa ferme en hôtel, mais se heurte à l'incompréhension de l'administration et à ses refus répétés. Hana se tourne alors vers un responsable influent du Parti qu'elle a connu comme garçonnet; ce dernier est directeur d'une grande laiterie et débloque la situation en faisant de l'hôtel en difficulté un foyer pour ses apprentis - comme quoi les rapports personnels et le Parti peuvent faire bon

56 Cité dans: Dietrich Scholze, Jurij Brězan Leben und Werk, Bautzen, Domowina-Verlag, 2016, p. 76.

57 Jurij Gros, Nach 20 Jahren nachgefragt, Bautzen, Lausitzer Druck- und Verlagshaus, 2009, p. 222-223.

58 Timo MešKAnK, Kultura w słužbje totalitarneho režima (La culture au service d'un régime totalitaire), Budyšin, Ludowe nakładnistwo Domowina, 2011, p. 105.

59 Jurij BrĚZAN, Bild des Vaters, Berlin, Verlag Neues Leben, 1982, p. 171.Édition sorabe:Zhromadźene Spisy, 10: Stary nan, Budyšin, Ludowe nakładnistwo Domowina, 1982, p. 166: «tón socialismus wšak njeje hubjeny, kusk jara wotře snano, ja měnju tak w nowinje a w telewiziji, a potom tež, dokelž chce wón wonhladać kaž tykanc, hdyž je chlěb tola tež něšto jara dobre. Ale štož so mi na tym socialismusu njelubi, je, zo so telko kradnje... na přikład tón Pětr ze sydlišća, tón wozy pěsk za kombinat, jedyn připowěšak sej wón za sebe sypnje, wón pěsk njetrjeba, jeho dom je hotowy, garaža tež. Dokelž wón pěsk wozy, kradnje wón pěsk. Wšitcy to wědźa, a nichtó ničo njepraji. Dokelž kóždy kradnje.»

60 Jurij KRawža, Ikarus pod módrym njebjom, Ludowe nakładnistwo Domowina, 1982, Kupa moja starosć (Mon lot, c'est mon affaire), p. 128-130. 
ménage par-delà l'administration ${ }^{(61)}$... Avec Jurij Krawža se dessine un conflit de générations dans l'exercice de la vie quotidienne. Face à une société très policée - dans tous les sens du terme - dans laquelle tout est prévu et codifié, l'imagination individuelle se réfugie dans le rêve, pour échapper à une réalité grise et industrieuse. On pense à une gravure du peintre Wolfgang Mattheuer (1927-2004), qui représente Icare, car une nouvelle donne son nom à tout le recueil paru en 1982: Ikarus pod módrym njebjom (Icare sur fond de ciel bleu). Dans cette première nouvelle, un fils docile aide son père à construire une maison familiale, construction en cours depuis des années. Le père appartient à la génération qui bâtit le pays, mais le projet ne séduit guère le fils, qui rêve de s'envoler depuis qu'il est tout petit. «Maćij, passe-moi le mortier!» ${ }^{(62)}$ crie le père juché sur l'échafaudage, mais le fils se fait prier, car il est ailleurs: il rêve de s'envoler comme Icare. La fin est amère, comme celle du héros antique; c'est dans sa vie personnelle et sentimentale que le jeune homme perdra toutes ses illusions. La grisaille reprendra le dessus, mais le garçon se promet de ne pas retomber...

Même si le roman tourne seulement autour d'amitiés déçues, la tonalité reste la même dans Landung der Träume (L'atterrissage des rêves) de Jurij Koch ${ }^{(63)}$.

Le héros d'une autre nouvelle de Jurij Krawža, General předsyda (Le général président, 1968), est lui aussi un rêveur qui rêve depuis toujours de parcourir les océans et de chasser les ours polaires, mais il se trouve à l'étroit dans le labeur quotidien d'une coopérative agricole qui, selon ses propres termes, «ne l'intéresse que dans la mesure où elle lui permet de gagner son pain... Jour après jour, j’attendais la fin de la journée de travail. Mais celle-ci terminée, je revivais mes rêves qui dansaient, virevoltaient, me pourchassaient que c'en était un délice ${ }^{(64)}$.

Le rêve en définitive pour échapper à une certaine réalité...

\section{Le regard rétrospectif}

La remise en cause de l'héritage social et culturel de la RDA au lendemain de la chute du Mur va en inquiéter plus d'un. Souvenons-nous des images de Potsdamer Platz lorsqu'il est question de faire jouer l'article 346 pour la réunification : «Kein Anschluß unter dieser Nummer!»- le jeu de mots renvoyant à une allusion politique difficilement traduisible.

Les écrivains sorabes partagent cette inquiétude, comme en témoignent ces réflexions de Jurij Brězan:

«Cela ne change rien au fait que les hommes continueront à rechercher une autre façon de vivre que celle où le profit est la valeur des valeurs» ${ }^{(65)}$.

61 Hany Mudrineje hotel (L'Hôtel de Hana Mudrina), in : ibid., p. 94-107.

62 J. KrawžA, Ikarus pod módrym njebjom (note 60), p. 10: «Maćijo, maltu!»

63 Jurij Koch, Landung der Träume (éd. allemande) et éd. sorabe: Nawrót sonow (Le Retour des rêves), Halle/Leipzig, Mitteldeutscher Verlag, 1982.

64 J. KRAwžA, General předsyda, in: Ikarus pod módrym njebjom (note 60), p. 20 : « kotrež mje jenož hišće telko zajimowaše, zo zasłužich sej z nim swój chlěb... a dźeń wote dnja čakach na swjatok. Po swjatoku wožiwjachu moje sony, rejowachu, skakotaju, honjachu mje, zo bě to radosć. »

65 Ingeborg SснміDт, «Suche nach dem Land Ohnefurcht... Gespräch mit Jurij Brězan», Leipziger Volkszeitung, 07.06.1991, cité dans D. Scholze, Jurij Brězan Leben und Werk (note 56), p. 271 : «Denn das ändert nichts daran, daß die Menschen weiter suchen werden nach einer anderen Art zu leben, als der, wo der Profit der Wert aller Werte ist. » 
Ou encore dans une lettre du 6 septembre 1990 adressée à une collègue française, où il exprime sa crainte:

«Il n’y aura pas d'Allemagne européenne, mais une Europe allemande» ${ }^{(66)}$.

Jurij Brězan avait publié en 1989 une autobiographie partielle, Mein Stück Zeit (Ma part de temps ${ }^{(67)}$. Étant donné la chute du Mur et les événements qui y étaient associés, le livre passa presque inaperçu. L'auteur rédigea une suite à ce premier volume avec Ohne Paß und Zoll. Aus meinem Schreiberleben (Sans Passeport ni Douane. Récits de ma Vie d'écriture) à la veille du nouveau millénaire ${ }^{(68)}$. Il consacre deux chapitres à l'Union des écrivains dont il a été vice-président pendant des années, et c'est l'occasion d'évoquer des souvenirs où apparaissent les grands du régime.

Walter Ulbricht, d'abord, et ses discours-fleuves lors du congrès de l'Union sur les mérites des auteurs d'œuvres pour la télévision - parmi lesquels se trouvent deux membres du Comité central, son refus de donner la parole à Stefan Heym, à qui il dit: «Si vous voulez aller dans le capitalisme, Monsieur Heym, je vous laisse y aller. Allez$y$, allez-y! ${ }^{(69)}$, et sa tentative d'exclure Jurij Brězan pour avoir soutenu un collègue rappelant le faible niveau des œuvres destinées à la télévision ${ }^{(70)}$; un collègue disparu depuis, dont l'écrivain taira le nom, qui a fait carrière grâce à la Stasi et est manœuvré par les puissants Ulbricht et Mielke pour évincer Brězan (p. 144-145);

Klaus Gysi, alors ministre de la Culture, et Erich Honecker, qui prennent à part notre écrivain, largement réélu à sa fonction de vice-président de l'Union des Écrivains, et décident de ne pas se mêler d'une affaire douteuse: Ulbricht n'a déjà plus le vent en poupe... (p. 145); Johannes Becher, poète officiel et ministre de la Culture, grand chasseur qui cherche toujours des partenaires et se laisse entraîner par des paysans rusés dans une affaire coûteuse où il se ridiculise ${ }^{(71)}$.

Brězan porte un jugement mitigé sur le jeune Heiner Müller, que le Parti lui a envoyé pour recadrer la Fête de la moisson, commande du ministère de la Culture de Saxe: «la couronne de moisson» et «le roi de la moisson» ont en effet été désapprouvés en tant «qu'héritage idéologique monarchiste» ${ }^{(72)} \ldots$

L'affaire Biermann a aussi laissé à Jurij Brězan un mauvais souvenir, car on a fait pression sur lui dans les instances du Parti pour l'amener à approuver et justifier l'exclusion et le retrait de citoyenneté du chanteur - ce qu'il a constamment refusé, déplorant les effets dévastateurs de la mesure à l'étranger ${ }^{(73)}$.

Mais Jurij Brězan ne se laisse pas aller au rejet global d'une époque:

66 Lettre de Jurij Brězan à Geneviève Cimaz à Paris. Cité dans: ibid., p. 272 : «Es [wird] kein europäisches Deutschland geben, sondern ein deutsches Europa.»

67 Jurij BRĚZAN, Mein Stück Zeit, Berlin, Verlag Neues Leben, 1989.

68 Jurij BrĚZAn, Ohne Paß und Zoll. Aus meinem Schreiberleben, Leipzig, Gustav Kiepenheuer Verlag, 1999.

69 Ibid., p. 140: «Wenn Sie in den Kapitalismus wollen, Herr Heym, ich lasse Sie gehen. Gehen Sie! Gehen Sie!»

70 Ibid., p. 141.

71 Ibid., p. 151-154.

72 Ibid., p. 161-162: «Ich schickte das Stück ab und bekam es zurück mit bösen Bemerkungen eines Landesparteisekretärs, der 'Erntekrone' und 'Erntekönig' als 'monarchistisches Gedankengut' befand. »

73 Ibid., p. 164-167. 
«Comme dernier point d'une somme toujours à faire, je considère cette époque à laquelle différents points de vue donnent des noms différents: après la fracture, après le changement, après l'unification. Leur jour numéro un, je ne l'ai pas ressenti comme un choc. Il était depuis longtemps prévisible qu'il viendrait, il fut décidé en 1986 à Reykjavik, où Gorbatchev face à Reagan - d'une autre manière que les Carthaginois face à Scipion - ne choisit pas la guerre. J'ai ressenti un choc seulement lorsque le capital engloutit le buffet de l'unification. Je souhaiterais bien ne pas avoir eu à inclure cela dans ma somme» ${ }^{(74)}$.

Dans une interview de juin 1991 par une journaliste de la Leipziger Volkszeitung, il fait remonter les choses encore plus loin: «Il est possible que ce soit le 17 juin qui ait joué un rôle important » ${ }^{(75)}$. Il n'avait fait allusion aux événements du 17 juin 1953 dans Mannesjahre que par le biais des craintes de deux anciens qui se remémoraient l'intervention de la Reichswehr trente ans auparavant ${ }^{(76)}$.

Dans un chapitre intitulé «Sekretäre», Jurij Brězan dresse le portrait d'autres personnages importants du régime. D’abord Hans Modrow, avec lequel il se liera d'amitié:

«Je n'ai pas besoin de présenter Hans Modrow, des livres l'ont décrit et il a écrit lui-même sur son rêve d'Allemagne et sa tentative à partir d'une réalité morcelée d'en forger une nouvelle. La tentative était honorable - et illusoire, c'était trop tard. Rien ne rendait le "trop tard" plus évident que les images de Dresde qui passaient à la télévision: le petit et frêle Hans Modrow à côté - plutôt: dans l'ombre - du colossal chancelier de Bonn et mille voix scandant en chœur "Helmut, Helmut" »" (77).

Puis Kurt Hager, la haute autorité du Comité central du Parti pour l'art et la culture:

«Bien sûr, je l'avais rencontré quelquefois... je l'avais entendu parler, j’avais lu ses articles, je le trouvais $\mathrm{sec}$, montrant peu de réelle compréhension pour l'art, par moment d'une étroitesse dogmatique et inébranlable - un homme - qui, même quand il disait des choses intelligentes, me concernait comme responsable, mais ne m’intéressait pas comme être humain» ${ }^{(78)}$.

74 Ibid., p. 174: «Als letztes zu der immer noch offenen Summe zähle ich jene Zeit, die von verschiedenen Standpunkten aus verschieden benannt wird: nach dem Umbruch, nach der Wende, nach der Vereinigung. Ihren Tag Eins habe ich nicht als Schock erlebt. Daß er kommen würde war lange absehbar, bestimmt wurde er 1986 in Reykiavik, wo Gorbatschow vor Reagan - anders als einst die Karthager vor Scipio - nicht den Krieg wählte. Wie ein Schock traf es mich erst, als das Kapital das Vereinigungsbuffet leerfraß. Ich wünschte sehr, ich hätte dieses nicht in meine Summe einbeziehen müssen. »

75 I. Sснмідт, «Suche nach dem Land Ohnefurcht... Gespräch mit Jurij Brězan» (note 65), p. 209 et note 152, p. 271 : «Möglicherweise ist es der 17. Juni, der eine gewichtige Rolle gespielt hat.»

76 J. BRĚZAN, Mannesjahre (note 45), p. 217-221.

77 J. BrĚZAN, Ohne Paß und Zoll (note 68), p. 179: «Hans Modrow brauche ich nicht darzustellen, er ist beschrieben worden in Büchern und hat selbst geschrieben über seinen Traum von Deutschland und seinen Versuch, aus einer zerbrochenen Wirklichkeit eine neue zu formen. Der Versuch war ehrenhaft - und illusorisch, es war zu spät. Nichts machte das “Zu spät” deutlicher als die Bilder, die aus Dresden über die Fernsehsender liefen: der schmächtige, nicht hochgewachsene Hans Modrow an der Seite - besser : im Schatten - des kolossalen Kanzlers aus Bonn und dazu ein tausendstimmiger skandierender Chor "Helmut, Helmut"."

78 Ibid., p. 181: «Natürlich war ich ihm manchmal begegnet... hatte ihn reden hören, seine Artikel gelesen, fand ihn trocken, mit wenig wirklichem Verständnis für Kunst, zuweilen von dogmatischer Enge und unbeweglich - ein Mann, der - auch wenn er Kluges von sich gab - mich anging als Funktionär, nicht aber interessierte als Mensch.» 
Avare de paroles et impénétrable, cet homme offre néanmoins à l'auteur lors de sa dernière rencontre un visage nouveau en mars 1989 et se livre à une confidence ${ }^{(79)}$ :

«Le socialisme est une expérimentation que nous avons nous-mêmes fait échouer, dit-il avec un étrange sourire perdu.»

Vient enfin Erich Honecker, avec qui l'écrivain avait toujours été en bons termes, et à qui il pose la question qui le préoccupe depuis 1969. À cette époque, un membre du Comité central avait proposé une nouvelle appellation de la Domowina, organisation nationale sorabe fondée en 1912, désormais qualifiée de «socialiste». La proposition avait été acceptée par tous les membres du présidium, puis votée à main levée. Un seul membre s'y était opposé: Jurij Brězan, qui dut payer son opposition de diverses brimades. L'introduction du terme dans les statuts entraîna nombre de défections, la population sorabe majoritairement rurale et christianisée associant le terme de socialiste à celui d'athée. De manière inattendue, Honecker reconnaît que la Domowina, pas plus que le Kulturbund ${ }^{(80)}$, n'est une organisation socialiste, mais qu'il convient de remplacer cet adjectif par un autre et l'écrivain de conclure:

«Allumfassend était aussi inoffensif que dépourvu de signification, il était content d'avoir trouvé le mot qu'il prononça plusieurs fois, hocha la tête et trouva que c'était ce qu'il fallait et que cela sonnait bien ${ }^{(81)}$.

Un souvenir particulièrement amer remonte à l'année 1956, lorsque l'écrivain fut exclu de la direction de la Domowina pour "passivité» dans la lutte pour la construction du socialisme. Il avait un ennemi dans le tribunal constitué par le présidium: Kurt Krjenc, un dogmatique, le futur Premier secrétaire de l'organisation ${ }^{(82)}$.

«Je les vis comme une part du pouvoir que, en Hongrie, en Pologne, le peuple déclarait coupable et eux, ils restaient sans visage derrière leur chaire et parlaient d'une seule voix: les hommes sont là pour le socialisme et non pas le socialisme pour les hommes ${ }^{\left({ }^{(3)}\right)}$.

C'est l'époque où il conçut l'idée du troisième volume de sa trilogie Felix Hanuš, qu'il publie et qui est promis à la distinction du Prix national. Il note: «Je crois que je n'ai écrit aucun livre avec plus de passion que celui-là - c'était un cri de colère en faveur de la raison» ${ }^{(84)}$. Mais il apprend qu'Ulbricht a mis son veto: «Les gens d'Ulbricht

79 Ibid., p. 184: «Der Sozialismus ist ein Experiment, das wir selbst scheitern ließen - sagte er mit einem sonderbaren, verlorenen Lächeln.»

80 Le Kulturbund était une «organisation de masse» selon la terminologie de la RDA, qui avait pour but la renaissance intellectuelle et morale du pays à partir de l'antifascisme et de l'humanisme; il était ouvert à tous.

81 Ibid., p. 188: «Allumfassend war so unschädlich wie unsinnig, er freute sich das Wort gefunden zu haben, sagte es ein paarmal vor sich hin, wiegte den Kopf und befand, es sei richtig und klinge auch gut.»

82 Cf. Maćica Serbska, Serbja pod stalinistiskim socializmom (Les Sorabes sous le socialisme stalinien), Procès verbal de la séance du 18.01.1992, Budyšin, Ludowe nakładnistwo Domowina, p. 87-88.

83 J. BrĚZAN, «Das Schreiben und das Geschriebene», in: Ohne Paß und Zoll (note 68), p. 195: «Ich aber sah sie als Teil der Macht, die in Ungarn, in Polen das Volk schuldig sprach, und sie standen hinter dem Katheder gesichtslos und sprachen aus einem Mund: die Menschen sind für den Sozialismus da und nicht der Sozialismus für die Menschen.»

84 Ibid., p. 197: "Ich glaube, ich habe kein Buch mit mehr Leidenschaft geschrieben als dieses - ein zorniger Aufschrei für Vernunft.» 
n'avaient pas manqué les quelques phrases défendant l'individu contre l'absolutisme du pouvoir ${ }^{\left({ }^{(8)}\right.}$. Il adresse alors une lettre incendiaire à la section Culture du Comité central, déclarant ne plus vouloir écrire en allemand, une décision sur laquelle il reviendra, bien sûr.

Jurij Brězan, qui avait eu les faveurs du régime, a le désir de clarifier sa position:

«Ce n'est pas parce que j’ai écrit il y a 42 ans un panégyrique de Staline - dont Churchill aussi faisait l'éloge, Staline dont les armées avait protégé sa Grande-Bretagne tout autant que mon petit peuple - mais parce que j'avais confié mes conceptions, passées par le filtre du vécu et de l'expérience, à une idéologie pour qu'elles se réalisent, et parce que je n'avais pas fait machine arrière, après avoir vu que les idéologies, plus elles s'institutionnalisent, et plus elles ne peuvent que se penser elles-mêmes... L'idéologie s'empara des idéaux et les déforma pour cacher le mal qui pourrissait l'intérieur en le présentant comme un mur protecteur contre les menaces extérieures. Mais cela ne fait pas des idéaux des invraisemblances. Le chrétien qui a connu les horreurs de l'Inquisition pouvait maudire et envoyer en enfer le cardinal inquisiteur et son armée d'esclaves, le sermon du Christ sur la montagne demeurait pour lui le fondement de la foi et un enseignement de vie ${ }^{\left({ }^{(8)}\right)}$.

Brězan connut le purgatoire après la disparition de la RDA. Il y fait lui-même allusion dans ses souvenirs ${ }^{(87)}$. Comme toujours dans ces périodes de bouleversements politiques, ce sont ceux que leurs fonctions ont mis à l'abri des tentations qui se font les juges des autres. Mais la stature de l'écrivain était trop grande pour qu'on puisse continuer à lui tourner le dos, d'autant plus qu'il s'était remis à écrire.

En 1990, la maison d'édition de la Domowina interroge à chaud les écrivains sur les événements. Lorsqu'on se tourne vers Jurij Grós, qui fut pendant 26 ans à la tête de la Domowina, député à la Chambre du Peuple et membre du SED - il a aussi été écrivain à ses heures -, et qu'on lui demande son avis sur le grand changement, il répond:

"La politique du SED et le vieux régime socialiste ont fait naufrage. Cette conception socialiste du Parti avec ses structures staliniennes se sont révélées fausses. Ce qui s'est passé maintenant dans notre pays a débuté en silence en 1985, lorsque la RDA n'a pas voulu mettre en pratique la perestroïka. Lénine a dit sur son lit de mort: quand un homme a trop de pouvoir en mains, cela tourne mal» ${ }^{(88)}$.

85 Ibid., p. 196: «Ulbrichts Leute hatten die wenigen Sätze der Verteidigung des Individuums gegen den Absolutismus der Macht nicht überlesen.»

86 Jurij BRĚZAN, Zwróćene dny, Spěšne zapiski serbskich awtorow (Jours de folie, Notes rapides d'auteurs sorabes), Budyšin, Ludowe nakładnistwo Domowina, 1991, p. 11-12: «Nic tohodla zo spisach před dwajaštyrceći lětami chwalospěw na Stalina - toho tež Churchill chwaleše, kotrehož Wulku Britanisku běchu Stalinowe armeje runje tak wuchowali kaž mój małki narod -, ale dokelž běch swoje předstawy, wufiltrowane $\mathrm{z}$ dožiwjeneho a nazhonjeneho, dowěrił ideologiji a njeběch to cofnył dowidźawši, zo móžeja ideologije, čim bóle so intitucionalizuja, čim bóle jeničce same sebje myslić... Idealow zmocowa so ideologija a deformowaše je na kryw za znutřka jědmjace zło, z tym zo jo jako škitnu murju přećiwo wohroženjam wotwonka wudawaše. To pak ideale njewěrne njesčini. Křesćan, kotryž bě hrózby inkwizicije dožiwił, móžeše kardinala inkwizicije a wójsko jeho wotročkow do hele zakliwać, Chrystusowe prědowanje na horje pak wosta jemu zasada wěry a žiwjenska wučba.»

87 J. BRĚZAn, «Dreimal: zu Hause», in: Ohne Paß und Zoll (note 68), p. 230-233.

88 Zwróćene dny, Ludowe nakładnistwo Domowina, Budyšin, 1991, Funkcionar organizacije (Le responsable de l'organisation), interview de Jurij Grós par Trudla Malinkowa le 2 juin 1990, p. 136: «Politika SED a stary socialistiski režim staj zwrěšćiłoj. Tuta socialistiska koncepcija strony z jeje 
Dans un ouvrage au titre significatif Što wot nas wostanje? (Qu'est-ce qui va rester de nous?) Křesćan Krawc recueille les souvenirs des paysans, qui décrivent les pressions qu'on leur a fait subir pour les amener à entrer dans les coopératives; l'un d'entre eux rappelle la très bonne récolte de pommes de terre en 1979 dans tout le canton. En tant que président de la coopérative, il prit alors l'initiative d'en vendre 1000 tonnes à la ville de Dresde pour éviter le gaspillage. Mais les autorités du canton le pénalisèrent pour ne pas leur avoir demandé l'autorisation de le faire, de sorte que ladite coopérative resta deux ans sans se voir attribuer de nouveau matériel ${ }^{(89)}$. On a là un exemple caractéristique de la gestion bureaucratique de la RDA.

Les écrivains qui avaient à fleuret moucheté stigmatisé la politique officielle d'extraction du lignite vont continuer à le faire, maintenant très ouvertement. Leurs critiques ne s'adressent plus désormais à la RDA, mais aux gouvernements des Länder, qui ont repris cette politique, ainsi qu'aux compagnies privées qui ont racheté la mine.

Dans Čertowa kowarnja (La Forge du Diable) Jurij Krawža décrit l'entreprise diabolique du magicien Woskura qui pollue et détruit la nature en exploitant les ressources du sous-sol. La fable va plus loin et constitue en fait une condamnation de la société industrielle productiviste, comme au temps de la $\mathrm{RDA}^{(90)}$.

Dans Jubel und Schmerz der Mandelkrähe (Joie et Chagrin du Rollier bleu) Jurij Koch fait une enquête de terrain et décrit le problème humain créé par l'avancée de la mine en interviewant ceux qui refusent de quitter le village, où ils ont passé leur vie, et ceux qui ont accepté le déracinement ${ }^{(91)}$. Il va même s'engager personnellement dans la lutte sur le terrain, et devenir un militant écologiste. Mais c'est la lutte inégale du pot de terre contre le pot de fer, car le lignite reste, dans l'Allemagne unifiée, ce qu'il était en RDA: la source d'énergie majeure du pays.

\section{Conclusion}

Les écrivains sorabes se sont trouvés en RDA dès les années 1960 dans une situation particulière. D'une part, ils se sentaient redevables envers un État qui avait reconnu leur identité, avait encouragé la création d'institutions culturelles qu'il soutenait financièrement et qui avait favorisé la promotion, la formation continue et la reconversion des diplômés tentés par la littérature et l'écriture: la majorité des écrivains sorabes sont d'origine paysanne et modeste. D’autre part, ils assistent à la dérive dogmatique et autoritaire d'un régime qui s'éloigne du socialisme qui les avait séduits au départ. Ils ne veulent visiblement pas prendre le risque de remettre en cause un statut favorable par des critiques trop acerbes qui pourraient leur valoir le reproche rédhibitoire

stalinististiskimi strukturami so njeje jako prawa wopokazała. To, štož je so w našim kraju nětkole stało, je so zaćichim zahajiło 1985, hdyž NDR njeje chcyła perestrojku přewjesć. Lenin je na smjertnym łožu prajił: Hdyž ma jedyn přewjele mocy w rukach, dźe to křiwje.»

89 Křesćan KRAwC, Što wot nas wostanje? (Qu'est-ce qui va rester de nous?), Budyšin, Ludowe nakładnistwo Domowina, 1991, Klučowe prašenje (La question-clé), p. 196.

90 Jurij KrawžA, Čertowa kowarnja (La Forge du Diable), Budyšin, Ludowe Nakładnistwo Domowina, 1993.

91 Jurij KocH, Jubel und Schmerz der Mandelkrähe (Joie et Chagrin du Rollier bleu), Bautzen, DomowinaVerlag, 1992. 
de nationalisme. Leur histoire leur a appris la modestie et la prudence, c'est ce qui explique le caractère modéré de leurs prises de position à l'égard du régime. C’est la RDA qui a assuré leur survie au lendemain du nazisme et de la guerre, c'est aussi grâce à la RDA que leur statut a été repris par la République fédérale lors de la réunification. Quitte à paraître comme d'éternels privilégiés, ils n'ont pas sombré après la chute du Mur dans la culpabilisation que certains voulaient leur infliger et se sont efforcés de rester lucides et conscients de leurs responsabilités envers leur peuple et leur culture. C'est ce qui leur a permis de continuer à exister et à produire, faisant dire que la seule chose qui a subsisté de la RDA, ce sont les Sorabes.

\section{Bibliographie}

\section{Jurij BRĚZAN}

Sorbische Erzählungen und Gedichte, Berlin, Verlag Volk und Welt, 1951.

Swět budźe rieńši (Le monde sera plus beau), Bautzen, Institut für sorbische Volksforschung, 1951.

Naš wšědny dźeń (Notre quotidien), Budyšin (Bautzen), Nakład Domowiny, 1955.

Mannesjahre (L'Âge d’homme), Berlin, Verlag Neues Leben, 1964. Edition en sorabe: Zrale lěta, Budyšin, Ludowe nakładnistwo Domowina, 1964.

Ansichten und Einsichten (Vues et Points de vue), Berlin, Verlag Neues Leben, 1976.

Bild des Vaters (L'Image du Père), Berlin, Verlag Neues Leben, 1982.

Erzählungen, Berlin, Verlag Neues Leben, 1987.

Mein Stück Zeit (Ma part de temps), Berlin, Verlag Neues Leben, 1989.

Ohne Paß und Zoll. Aus meinem Schreiberleben (Sans passeport ni douane. Scènes de ma vie d'écriture), Leipzig, Gustav Kiepenheuer Verlag, 1999.

Zhromadźene spisy (CEuvres complètes), Budyšin, Ludowe nakładnistwo Domowina, 19651998. Édition préparée jusqu'en 1997 par Pawoł Vőlkel, t. 13 , annotée et commentée par Lucija Hajnec en 1998.

\section{Jurij Grós}

Staatsangehörigkeit: Deutsch, Nationalität: Sorbe (Citoyenneté: Allemand, nationalité: Sorabe), Schkeuditz, GNN Verlag, 2004.

Nach 20 Jahren nachgefragt (Questions posées 20 ans après), Bautzen, Lausitzer Druck- und Verlagshaus, 2009.

Joachim KeIL, Betrachtungen zum Werk Jurij Brězans (Considérations à propos de l'œuvre de Jurij Brězan), Bautzen, VEB Domowina-Verlag, 1976.

\section{Jurij KocH}

Mjez sydom mostami (Entre sept ponts), Budyšin, Ludowe nakładnistwo Domowina, 1968.

Róžamarja, Budyšin, Ludowe Nakładnistwo Domowina, 1975.

Landvermesser (Géomètres), in: Der einsame Nepomuk, Halle/Leipzig, Mitteldeutscher Verlag, 1978. En sorabe: Kotjatkowa dźiwna lubosć (L'étrange Amour de Kotjatko), in: Wosamoćeny Nepomuk, Budyšin, Ludowe nakładnistwo Domowina, 1978.

Landung der Träume. Éd. sorabe: Nawrót sonow (Le Retour des rêves), Halle/Leipzig, Mitteldeutscher Verlag, 1982.

Der Kirschbaum (Le Cerisier), Halle/Leipzig, Mitteldeutscher Verlag, 1984. Édition en sorabe: Wišnina, Budyšin, Ludowe nakładnistwo Domowina, 1984.

Jubel und Schmerz der Mandelkrähe (Joies et Chagrins du Rollier bleu), Bautzen, Domowina Verlag, 1992. 


\section{Křesćan KRAWC}

W delanach na Katyrnu (Dans le Plat-pays à la Sainte Catherine), Ludowe nakładnistwo Domowina, 1979.

Wočakńmy nalěćo (Attendons le printemps), Ludowe nakładnistwo Domowina, Budyšin, 1989.

Što wot nas wostanje? (Qu'est-ce qui va rester de nous?), Budyšin, Ludowe nakładnistwo Domowina, 1991.

\section{Jurij KRAWŽA}

Ikarus pod módrym njebjom (Icare sur fond de ciel bleu), Ludowe nakładnistwo Domowina, 1982. Čertowa kowarnja (La forge du Diable), Budyšin, Ludowe nakładnistwo Domowina, 1993.

MaćICA SER BSKA, Serbja pod Stalinistiskim Socializmom 1945-1960 (Les Sorabes sous le Socialisme stalinien), Budyšin, Ludowe nakładnistwo Domowina, 1992 / Protokol schadźowanja Maćicy, Serbskeje.

Kito LoRenc, SP Serbska poezija, Budyšin, Ludowe nakładnistwo Domowina, 1979.

Timo MešKank, Kultura $w$ słužbje totalitarneho režima (La Culture au service d'un Régime totalitaire), Budyšin, Ludowe nakładnistwo Domowina, 2011.

Edmund Pech, Die Sorbenpolitik der DDR 1949-1970 (La politique sorabe de la RDA 19491970), Bautzen, Domowina-Verlag, 1999.

Edmund Pесн \& Dietrich Scholze, Zwischen Zwang und Beistand (Entre Contrainte et Assistance), Bautzen, Domowina-Verlag, 2003.

Dietrich Scholze, Jurij Brězan Leben und Werk (Jurij Brězan. La Vie et l'CEuvre), Bautzen, Domowina-Verlag, 2016.

Angela Stachowa, Vineta, Budyšin, Ludowe nakładnistwo Domowina, 1983.

Měrćin VöLKeL

Přinoški k stawiznam serbskeho pismowstwa lět 1945-1990 Zběrnik (Recueil d'articles sur la littérature sorabe de 1945 à 1990), Budyšin, Ludowe nakładnistwo Domowina, 1994.

Zwróćene dny Spěšne zapiski serbskich awtorow (Jours de folie. Notes rapides d'auteurs sorabes), Budyšin, Ludowe nakładnistwo Domowina, 1991.

\section{Zusammenfassung}

Es sollte lehrreich sein nachzusehen, wie die sorbischen Schriftsteller die DDR wahrgenommen haben, da die Sorben in diesem Staate die einzige ethnische Minderheit bildeten; man darf also annnehmen, dass die angeführten Meinungen, Standpunkte, Bemerkungen oder Andeutungen der sorbischen Bevölkerung zuzumuten sind. Wie die sorbischen Schriftsteller zur DDR standen änderte sich aber im Lauf der 40 Jahre des Staates. In den Jahren, die dem Zusammenbruch folgten, standen sie hinter einem Regime, das den Sorben mehrere kulturelle Institutionen gewährte, die sie vorher nie gekannt hatten. Je mehr immerhin die Regierung auf Methoden zurückgriff, die demokratischen Praktiken fremd waren, desto mehr distanzierten sich die Schriftsteller von der Macht. Sie lernten ihre Sprache zu verkleiden und Berichte und Erzählungen hinter der Parabel oder dem Humor zu maskieren. Ab 1990 sprachen sie alles aus, was sie bis dahin verschwiegen hatten. Ihre Kritiken blieben jedoch gemäßigt, denn sie hatten vor, einen günstigen Status nie in Frage zu stellen, der den Sorben zum erstenmal in der Geschichte gerade durch die DDR zugesichert worden war. 


\begin{abstract}
It might be interesting to examine the attitude of the Sorbian writers towards the GDR, since the Sorbs were the only ethnic minority of the country and one can assume that their writings reflect the approach of the Sorbian population. That attitude evolved during the 40 years of the GDR's existence. At the beginning, soon after the end of the Second World War, the Sorbian writers supported a new regime, that granted the Sorbians many cultural institutions, a privilege they had not known before. But as the regime became more and more authoritarian, the Sorbians were progressively faced with measures far from a democratic practice. The writers learned to mask their remarks, for caution was recommended. From 1990 on, the writers expressed freely what they had had previously to keep for themselves. However, those critics were always moderate, because the Sorbian writers never wanted to jeopardise a status the Sorbian population had been granted by the GDR for the first time in History.
\end{abstract}

